\title{
Three-dimensional Printing of Bone Extracellular Matrix for Craniofacial \\ Regeneration
}

Ben P. Hung ${ }^{1,2}$, Bilal A. Naved ${ }^{4}$, Ethan L. Nyberg ${ }^{1,2}$, Miguel Dias ${ }^{1,2}$, Christina A. Holmes ${ }^{3}$, Jennifer H. Elisseeff $^{1,2}$, Amir H. Dorafshar ${ }^{5 *}$, and Warren L. Grayson ${ }^{1,2 *}$

1 Department of Biomedical Engineering, The Johns Hopkins University School of Medicine, Baltimore, MD, USA

2 Translational Tissue Engineering Center, The Johns Hopkins University School of Medicine, Baltimore, MD, USA

3 Department of Neurosurgery, The Johns Hopkins Hospital, Baltimore, MD, USA

4 Fischell Department of Biomedical Engineering, University of Maryland, College Park, MD, USA

5 Department of Plastic Surgery, The Johns Hopkins Hospital, Baltimore, MD, USA

${ }^{*}$ Corresponding authors:

Warren L. Grayson, PhD

400 N. Broadway, Smith 5023

Baltimore, MD 21231

Tel: 410-502-6306; Fax: 410-502-6308

E-mail: wgrayson@jhmi.edu

Amir H. Dorafshar, MBChB

601 N. Caroline St.

Baltimore, MD 21231

Email: adorafs1@jhmi.edu

This supplementary information documents contains 4 pages and 2 figures. 


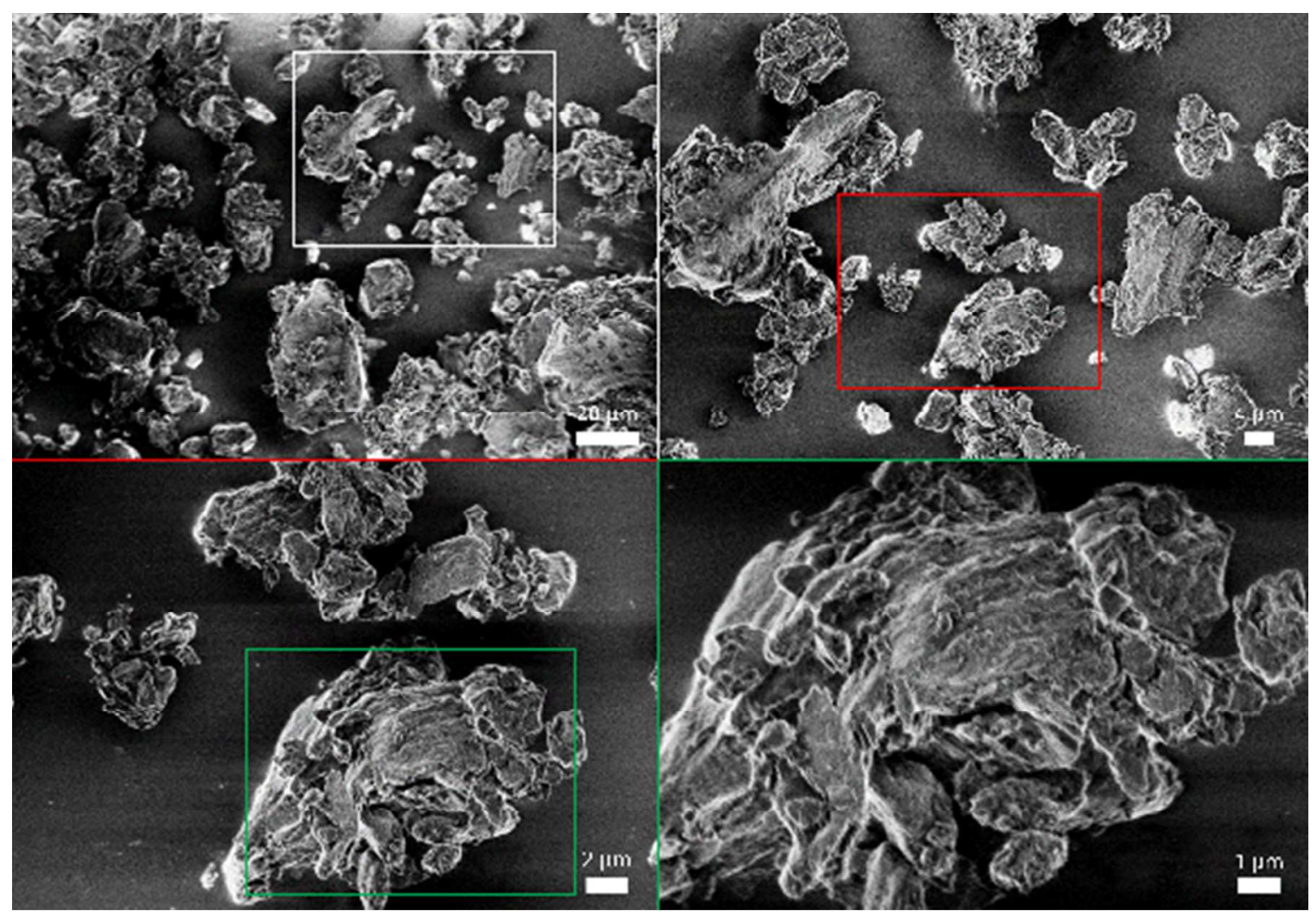

Supplementary Figure S1 


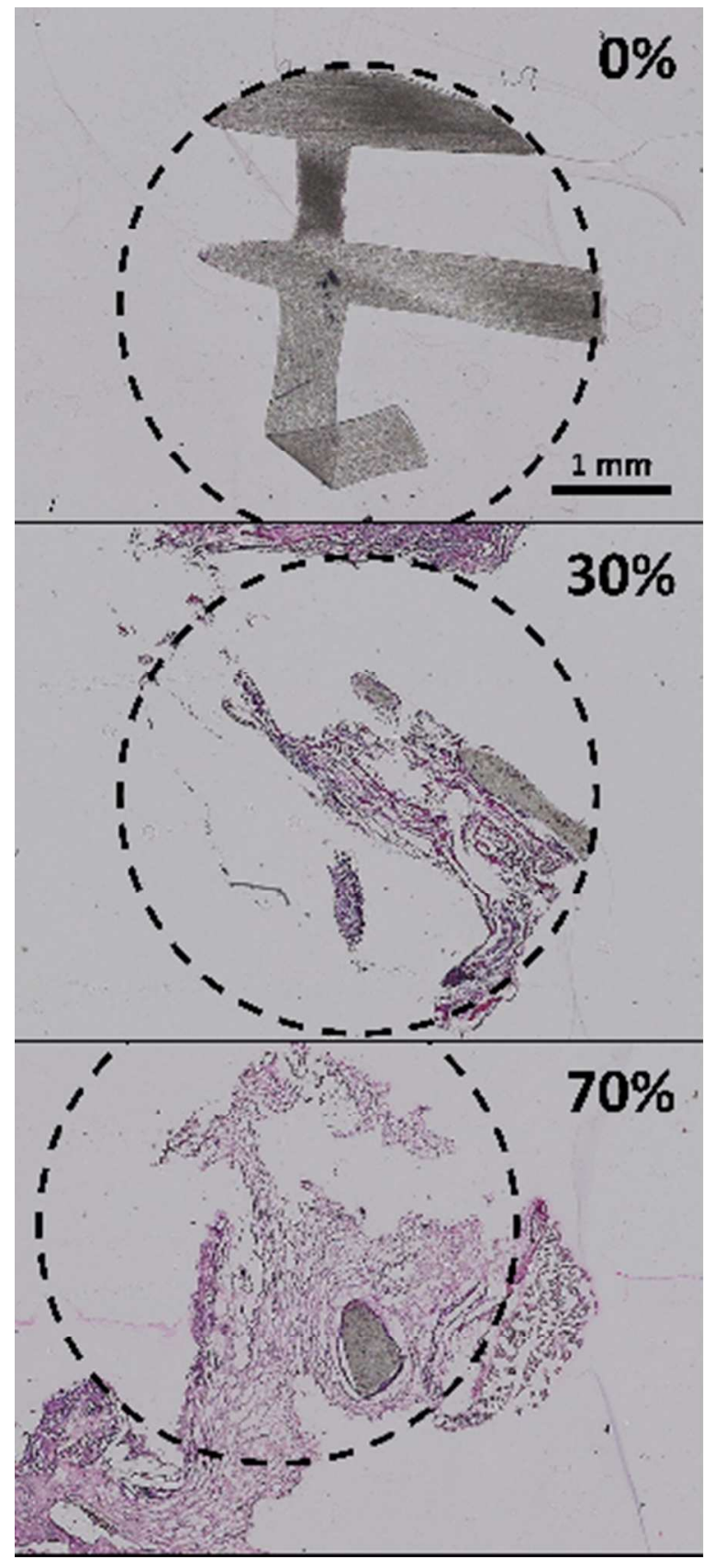

Supplementary Figure S2 
Supplemental Figure S1: Scanning electron microscopy of cryo-milled bone particles that had been run through a $40 \mu \mathrm{m}$ filter. Sizes of particles above $40 \mu \mathrm{m}$ are absent. Colored boxes show images of increased magnification.

Supplemental Figure S2: In vivo host cell infiltration into pure and hybrid scaffolds one week post-implantation. Black dotted lines show boundaries of scaffolds while the haematoxylin/eosin stain shows infiltration of host cells. Infiltration is negligible in the pure scaffolds and increases with increasing concentration of bone in the hybrid scaffolds. 